\title{
IJIMI
}

International Journal of Machine Intelligence

ISSN: 0975-2927 \& E-ISSN: 0975-9166, Volume 3, Issue 3, 2011, pp-134-137

Available online at http://www.bioinfo.in/contents.php?id=31

\section{AN EFFICIENT METHOD OF VEHICLE NUMBER PLATE DETECTION AND RECOGNITION}

\author{
ANISH LAZRUS ${ }^{1}$, SIDDHARTHA CHOUBEY ${ }^{2}$, SINHA G.R. ${ }^{3}$ \\ 'Department of Computer Science, Faculty of Engg. \& Technology, Shri Shankaracharya Group of Institutions, Bhilai, INDIA \\ 2Department of Computer Science, Faculty of Engg. \& Technology, Shri Shankaracharya Group of Institutions, Bhilai, INDIA \\ ${ }^{3}$ Faculty of Engineering and Technology, Shri Shankaracharya Group of Institutions, Bhilai, INDIA \\ *Corresponding Author: Email- anishlazrus@gmail.com, sidd25876@gmail.com, drgrsinha@ieee.org
}

Received: September 29, 2011; Accepted: November 03, 2011

\begin{abstract}
License plate location is an important phase in vehicle license plate recognition for intelligent transport systems. The objective of this work is to design and implement an efficient method for License Plate Recognition (LPR) of Indian License Plates. This paper presents a robust method of license plate location, segmentation and reorganization of the characters present in the located plate. The images of various vehicles have been acquired manually and converted in to gray-scale images. Then wiener2 filter is used to remove noise present in the plates. The segmentation of gray scale image generated by finding edges using Sobel filter for smoothing image is used to reduce the number of connected component and then bwlabel is used to calculate the connected component. Finally, single character is detected. The results show that the proposed method achieved accuracy of $98 \%$ by optimizing various parameters with higher recognition rate than the traditional methods
\end{abstract}

Keywords - license plate, recognition, segmentation, noise, and filter.

\section{Introduction}

License Plate Recognition (LPR) is an image processing technology used to identify vehicles by their license plates. LPR is one form of Intelligent Transport Systems (ITS) technology that not only recognizes and counts vehicles [1] but distinguishes each as unique. With the wide use of computing technology, Intelligent Transportation System becomes more and more important in Traffic management [2]. A LPR system can be conceptually considered as containing two separate processing stages:

- $\quad$ License Plate Localization (LPL);

- $\quad$ License Plate Character Recognition (LPCR). License plate localization is an important phase in vehicle license plate recognition of intelligent transport systems. It can be used in many applications such as entrance check, security, and parking control, airport or harbor cargo control, road traffic control, and speed control and so on. A number of commercial software is developed in this area.

However, sets of blurry and skewed snapshots give worse recognition rates than a set of snapshots, which has been captured clearly [3]. Due to the rapidly increase in number of vehicles across the world's big cities, vehicle number plate recognition system has become one of the most important digital image processing systems to be used [4]. This system will solve so many problems for these city facilities which is hard to be controlled by human 24 hours. However, this system is still facing a lot of difficulties to be able to recognize license plates and one of these difficulties is the large variety of plate's layouts between the different countries and even across the same country cities [5]. This work tends to find an integrated System which can recognize these license plates automatically in real-time applications.

\section{Related work}

In this paper, a method is proposed for detecting the license plate form a grayscale image and segment the characters contained by the License plate of a car. For real time application, the system requires a video camera which acquires the image of vehicles from rear or front [7] but for the present work, due to unavailability of the required hardware, we have used mobile camera. The image of various parked vehicle have been acquired manually [8] thereafter fed to the software where they are first converted into grayscale image and then the grayscale image is segmented from grayscale image and then the region with highest probability of number plate, the single character is detected. This efficient method is used to capture characteristics of License plate in the image regions. Fig. (1) Depicts the flow diagram of the proposed work. A good number of plates are being localized so that to perform segmentation and recognition of License Plates (LP) [9]. The first processing stage deals with locating, even approximately, the position of the LP within the image under consideration [10]. We refer to this stage as acquire image, locating the LP amounts to identify the sub-image containing the full LP 
with as little extra background as possible [11]. This localization step is necessary, because it greatly reduces the complexity of locating and, subsequently, segmenting the characters that make up the LP [12]. Fig. (2) shows the input image acquired. Its gray scale image is shown in Fig. (3).

\section{Methods and Implementation}

The implementation takes place following the sequence of steps listed below:

- The recognition system has a PC which captures the vehicle license plate.

- Under adverse environmental conditions which refer to the following point below which make vehicle license plate images unclear when it is captured by the system:

1. poor Lighting and low contrast due to overexpose, reflection or shadows;

2. unforeseen weather like raining or haze;

3. blurry images;

4. dimming the lighting of the image; and

5. adding Salt and Pepper to the image.

- The system will identify the vehicle license plate and convert the images to grayscale images. Following by converting the grayscale images to binary images which consist of only ' 0 ' and ' 1 [13].

- After binary images, the system will do a character segmentation of the vehicle license plate. It will segment the character and number to each individual figure.

- All the character and number will then be change to binary form in terms of matrix and been recognize by neural network [14].

- A Graphical User Interface (GUI) will be use to display the process of the vehicle license plate been capture. Following by image cropping and recognition.

- The objective of second stage is to convert the input image in to gray scale image using rgb2gray conversion. Then the wiener2 filter is used to remove the noise from grayscale image. Wiener 2 low pass filter denoises a grayscale image that has been degraded by constant power additive noise [15]. It uses a pixel wise adaptive Wiener method based on statistics estimated from a local neighborhood of each pixel [16]. $\mathrm{J}=$ wiener2 (I, [m n], noise) filters the image I using pixel wise adaptive Wiener filtering, using neighbor hoods of size m-by-n to estimate the local image mean and standard deviation If we ignore the [m $\mathrm{n}]$ argument, $\mathrm{m}$ and $n$ default to 3 . The additive noise (Gaussian white noise) power is assumed to be noise. [J, noise $]=$ wiener2 (l, [m n]) also estimates the additive noise power before doing the filtering. Wiener2 returns this estimate in noise. Algorithm for noise removal wiener2 estimates the local mean and variance around each pixel. $\mu=\frac{1}{N M} \sum_{n 1, n 2 \in n} a(n 1, n 2)$

(1)

- $\sigma^{2}=\frac{1}{N M} \sum_{n 1 n 2 E n} a^{2}(n 1, n 2)-\mu^{2}$ (2)

- Where $\eta$ is the $N$-by-M local neighborhood of each pixel in the image A. wiener2 then creates a pixel wise Wiener filter using these estimates, Equation 1 and Equation 2.

- $\mathrm{b}(\mathrm{n} 1, \mathrm{n} 2)=\mu+\frac{\sigma^{2}-v^{2}}{\sigma^{2}}(\mathrm{a}(\mathrm{n} 1, \mathrm{n} 2)-\mu$ (3) Where v2 is the noise variance in equation 3. If the noise variance is not given, Wiener2 uses the average of all the local estimated variances.

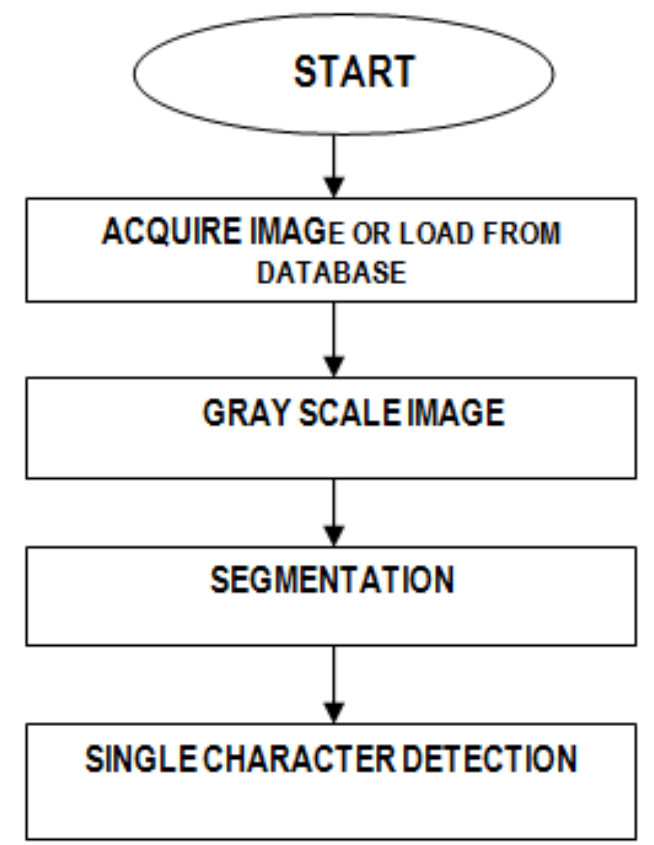

Fig. 1- The flow diagram for proposed system

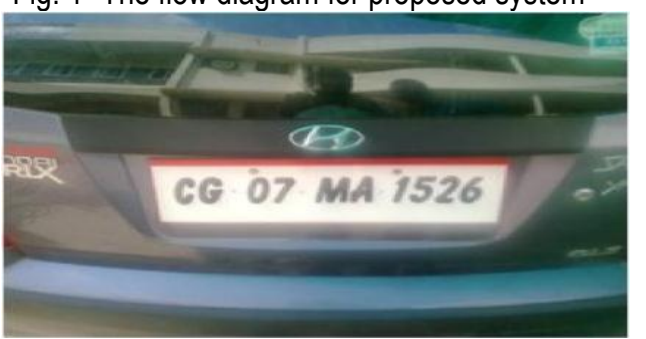

Fig. 2- Acquired input image

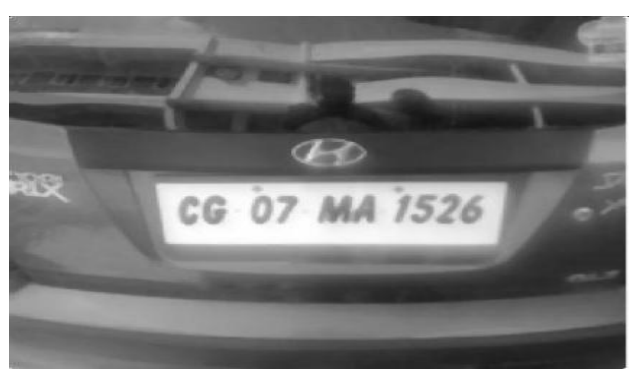

Fig. 3- Gray scale image 


\section{Segmentation and Result}

For the segmentation and detection of characters for recognition, we used Sobel filter to find the edges. Typically, a segmentation method is used to separate the pixels constituting the LP character from the background pixels [19]. The result of segmentation is shown in Fig. (4).

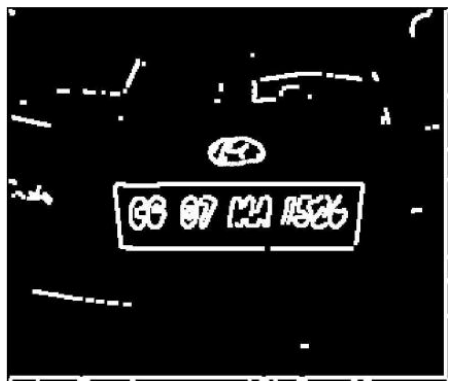

Fig. 4- Segmented image

After vehicle features have been extracted from the input images, feature segmentation is performed to separate individual elements according to the type of part or feature. In the case of vehicle number plates, the Chinese and English characters, numbers are separated to form a single character, alphabet or number [20]. Now this set is ready to be presented to the neural network recognizing the characters, alphabets, and numbers [21]. Single character detection has been shown in Fig. (5).The main objective of this paper is to provide an input image of license plate that has been extracted from an image of real scene using mobile camera. Then the characters and numbers are segmented and recognized. This process has covered all forms of Indian license plates. Table 1 shows recognition accuracy rate on real scene sample.

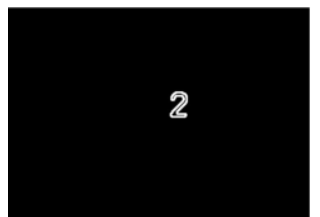

Fig. 5-Single character detection

TABLE 1: RECOGNITION ACCURACY RATE ON REAL SCENE SAMPLE:

\begin{tabular}{|c|c|c|c|c|c|}
\hline $\begin{array}{l}\text { S. } \\
\text { N. }\end{array}$ & $\begin{array}{l}\text { Research } \\
\text { papers }\end{array}$ & $\begin{array}{l}\text { Real } \\
\text { time } \\
\text { data }\end{array}$ & $\begin{array}{l}\text { Images } \\
\text { correctly } \\
\text { detected }\end{array}$ & $\begin{array}{l}\text { character } \\
\text { known }\end{array}$ & Results \\
\hline 1 & $\begin{array}{l}\text { Kok Kiaw } \\
\text { T et al. } \\
(2003)\end{array}$ & 60 & 49 & 50 & 83 \% \\
\hline 2 & $\begin{array}{c}\text { F.Martin } \\
\text { et al } \\
\text { (2002) }\end{array}$ & 75 & 67 & 66 & $88 \%$ \\
\hline 3 & $\begin{array}{c}\text { Proposed } \\
\text { Method }\end{array}$ & 50 & 46 & 49 & $98 \%$ \\
\hline
\end{tabular}

Most of the effort was spent on image processing methods like thresholding, segmentation and feature extraction which are crucial for the recognition system [22]. Great amount of time were spent on training the correlation in two dimensions and building up the templates for the classification [23]. Overall the vehicle license plate recognition software has been successfully designed and developed to recognize the 38 different characters using correlation in two dimensions. As results achieved are not $100 \%$ accurate, there are some methods that would enhance efficiency and reliability of the program for future work.

\section{Conclusions and future scope}

The process of vehicle number plate recognition requires a very high degree of accuracy when we are working on a very busy road or parking which may not be possible manually as a human being tends to get fatigued due to monotonous nature of the job and they cannot keep track of the vehicles when there are multiple vehicles are passing in a very short time [24]. To overcome this problem, many efforts have been made by the researchers across the globe for last many years. A similar effort has been made in this work to develop an accurate and automatic number plate recognition system. We have used neural-network and fuzzy logic in MATLAB to obtain the desired results. The setup has been tested for 50 vehicles containing different number plates from different states. In the process of final evaluation after optimizing the parameters like brightness, contrast and gamma, adjustments, optimum values for lightening and the angle from which the image is to be taken. We get an overall efficiency of $98 \%$ for this system. Though this accuracy is not acceptable in general, but still the system can be used for vehicle identification. It may be concluded that the project has been by and far successful. It can give us a relative advantage of data acquisition and online warning in case of stolen vehicles which is not possible by traditional man handled check posts. While thousands of vehicles pass in a day.

Though we have achieved an accuracy of $98 \%$ by optimizing various parameters, it is required that for the task as sensitive as tracking stolen vehicles and monitoring vehicles for homeland security an accuracy of $100 \%$ cannot be compromised with. Therefore to achieve this, further optimization is required. Also, the issues like stains, smudges, blurred regions \& different font style and sizes are need to be taken care of.

\section{References}

[1] Siddartha Choubey and Sinha G.R. (2011) Pixel Distribution Density based character recognition For Vehicle License Plate, (ICNCS 2011), 5, 26-30.

[2] Siddhartha Choubey, Sinha G.R., Bhagwati Charan Patel, Abha Choubey and Kavita Thakur (2011) 2011 3rd International Conference on Machine Learning and Computing(ICMLC 2011, Singapore, 4, , 390394.

[3] Ondrej Martinsky (2007) Algorithm and mathematical principles of automatic number plate recognition systems, B.Sc.-Thesis, BRNO University of Technology.

[4] Lee J.C.M., Wong W.K., Fong H.S (1999) IEEE, 2824-2828. 
[5] Shridhar M., Miller J.W.V.,Houle G., Bijnagte L. (1999) Proceedings of the Fifth International Conference on Document Analysis and Recognition, $17-20$.

[6] Haris Al-Qodri Maarif and Sar Sardy (2006) Proceedings of siding Fifth International Conference on image processing, 176-182.

[7] Akoum A., Daya B., Chauvet P. (2009) Journal of Theoretical and Applied Information Technology, 25-32.

[8] Koval V. (2003) IEEE International Workshop on Intelligent Data Acquisition and Advanced Computing Systems: Technology and Applications, Ukraine, 123-127.

[9] Christos Nikolaos (2008) IEEE Transactions on Intelligent transportation systems, 9, 377-391.

[10] Michael Seul, Practical Algorithms for Image Analysis Description, Examples and Code.

[11] Wisam Al Faqheri (2009) International Journal of Computer Science and Network Security, 9, 333-340.

[12] Nelson Kennedy Babu C. (2008) International Journal of Imaging Science and Engineering (IJISE), 2. 189-194.

[13] Kroese B. (1996) An Introduction to Neural Networks, Amsterdam University of Amsterdam,.

[14] Stephen Chapman J. (2002) MATLAB Programming for Engineers, 2nd Edition, Brooks/Cole Publishing Company.

[15] Hagan M. T., Demuth H. B. and Beale M. H. (1996) Neural Network Design, PWS Publishing, Boston.

[16] Jae Lim S. (1990) Two-Dimensional Signal and Image Processing, Prentice Hall, 469-476.

[17] Kok Kiaw Teo, (2003) Low Cost Number Plate Recognition, University of Queensland, Queensland.

[18] Parker J.R., Federl P. (1996) An approach to license plate recognition, Computer Science Technical Reports, University of Calgary.
[19] Martín F., García M. and Alba J.L. (2002) International Conference on Signal Processing, Pattern Recognition and Applications, Greece.

[20] Yazid M. Sharaiha, Binary Digital Image Processing.

[21] Etter D.M. (2008) Introduction to MATLAB, $2^{\text {nd }}$ edition.

[22] Humayun Karim Sulehria (2008) Electronics and Information Engineering, Issue , 7(6). 781790.

[23] Sivanandam S.N., Introduction to Neural Networks using MATLAB 6.0.

[24] Alasdair Mcandrew, Introduction to Digital Image Processing.

[25] John C. Russ. The Image Processing Handbook, fifth edition. 